\title{
MODERN APPLICATIONS OF COVARIANT DENSITY FUNCTIONAL THEORY*
}

\author{
P. Ring ${ }^{1}$, H. Abusara ${ }^{2}$, A. V. Afanasjev ${ }^{2}$, G. A. Lalazissis ${ }^{3}$, T. Nikšić ${ }^{4}$, and D. Vretenar ${ }^{4}$ \\ ${ }^{1}$ Physik-Department der Technischen Universität München, D-85748 Garching, Germany \\ ${ }^{2}$ Department of Physics and Astronomy, Mississippi State University, MS 39762 \\ 3 Department of Theoretical Physics, Aristotle University Thessaloniki, GR-54124, Greece \\ ${ }^{4}$ Physics Department, Faculty of Science, University of Zagreb, 10000 Zagreb, Croatia
}

\begin{abstract}
Modern applications of Covariant Density Functional Theory (CDFT) are discussed. First we show a systematic investigation of fission barriers in actinide nuclei within constraint relativistic mean field theory allowing for triaxial deformations. In the second part we discuss a microscopic theory of quantum phase transitions (QPT) based on the relativistic generator coordinate method.
\end{abstract}

\section{Introduction}

Theoretical approaches based on Density Functional Theory (DFT) remain undoubtedly among the most successful microscopic descriptions of nuclear structure ${ }^{1}$. One of the most successful schemes of this type is covariant density functional theory $(\mathrm{CDFT})^{2 \mid 3}$. It is based on Lorentz invariance, connecting in a consistent way the spin and spatial degrees of freedom in the nucleus. Therefore, it needs only a relatively small number of parameters which are adjusted to reproduce a set of bulk properties of finite nuclei and it is valid over the entire periodic table.

Over the years, Relativistic Mean-Field (RMF) models based on the CDFT have been successfully applied to describe ground state properties of finite spherical and deformed nuclei over the entire nuclear chart. Excited states have been investigated in the framework of relativistic RPA and the relativistic Cranking mode 3 . Here we restrict ourselves to two more recent applications of this concept. In section 2 we discuss a systematic investigation of the inner fission barriers in the actinide region and in section 3 we show an application of the relativistic Generator Coordinate Method (GCM). It allows a microscopic description of quantum phase transitions in finite nuclei, which have been investigated extensively in recent years by phenomenological models.

\section{Triaxial fission barriers in the actinide region.}

Most of the investigations of fission barriers have been carried out in the framework of non-relativistic models 4 . A study of the inner fission barrier heights $B_{f}^{s t}$ of

*ring@ph.tum.de 
even-even nuclei is motivated by the importance of this quantity for several physical phenomena. Many heavy nuclei decay by spontaneous fission, and the size of the fission barrier is a measure for the stability of a nucleus reflected in the spontaneous

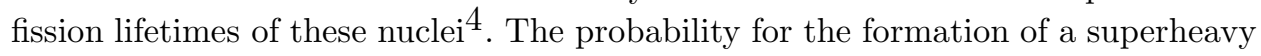
nucleus in a heavy-ion-fusion reaction is also directly connected to the height of its fission barrier. $B_{f}^{s t}$ is a decisive quantity in the competition between neutron evaporation and fission of a compound nucleus in the process of its cooling. The large sensitivity of the cross section $\sigma$ for the synthesis of the fissioning nuclei on the barrier height $B_{f}^{s t}$ stresses the need for accurate calculations of this value. For example, a change of $B_{f}^{s t}$ by $1 \mathrm{MeV}$ changes the calculated survival probability of a synthesized nucleus by about one order of magnitude or even more. The population and survival of hyperdeformed states at high spin also depends on the fission barriers. In addition, the fission barriers of very neutron-rich nuclei have (among other quantities such as Q-values or $\beta$-decay rates) an influence on the $r$-process of stellar nucleosynthesis.

During the last decade the role of triaxiality in the region of the saddle point of fission barriers has been recognized and tested in many non-relativistic frameworks. It was found that the height of the barrier is reduced when triaxial shapes are allowed $\sqrt[516]{ }$. However, this lowering strongly depends on the proton and neutron numbers and on the model employed. Investigations of inner fission barriers with triaxiality included are available within the frameworks of the microscopic + macroscopic method, the extended Thomas-Fermi plus Strutinsky integral, and non-relativistic energy density functionals based on Skyrme and Gogny forces.

Covariant density functional theory $2 \sqrt{2 / 3}$ is an approach alternative to the above mentioned non-relativistic methods. The progress in the study of the fission barriers within CDFT has been slower than in its non-relativistic counterparts. Inner fission barriers in several nuclei have been calculated mostly in the axially symmetric relativistic mean field (RMF) + BCS approach. Many of these investigations use the constant gap approximation in the BCS part. However, our recent study of various pairing schemes 7 used for calculations of fission barriers clearly shows that this approximation leads to unphysical results, because the gap changes considerably along the fission path. Only relatively few fission barriers have been studied in axially symmetric RMF calculations within the BCS approximation using an effective density-dependent zero-range force in the pairing channel presenting a much more realistic approximation for the pairing channe 8977 .

Unfortunately, axially symmetric calculations cannot be directly compared with experimental data since, as has been shown in non-relativistic calculations 5 , the lowering of fission barriers due to triaxiality is significant and can reach 3-4 MeV in some nuclei. At present, no systematic studies of the effects of triaxial degrees of freedom on the height of inner fission barriers are available in covariant density functional theory. Here we discuss recent systematic investigations 10 of the inner fission barriers within the triaxial RMF + BCS approach, and confront these CDFT results with experimental quantities as far as possible. 




Fig. 1. (Color online) Potential energy surface in ${ }^{240} \mathrm{Pu}$. The energy difference between two neighboring equipotential lines is equal to $0.5 \mathrm{MeV}$. The blue dashed line with solid circles shows the lowest in energy solution as a function of $\beta_{2}$. Further details are given in the text.

In this investigation we use the parameter set NL3*11 which is a modern version of the famous set NL3 and we employ in the pairing channel a monopole force with a set of coupling constants $G(N, Z)$ adjusted carefully to experimental odd-even mass differences in this region 10 . In Fig. 1 we show as an example the potential energy surface of the nucleus ${ }^{240} \mathrm{Pu}$ in the $\beta-\gamma$ plane. For axial symmetry we find the normal deformed minimum of the ground state at a deformation $\beta \sim 0.28$, a maximum at $\beta \sim 0.52$ and a superdeformed minimum at $\beta \sim 0.96$. We observe that the fission path (the blue dashed curve) bypasses the axial barrier between the normal and superdeformed minima. The barrier height is determined by the maximum of the energy along this fission path.

The deformation energy curves for other even-even nuclei in this region obtained in these calculations are shown in Fig. 2. Full black curves show axially symmetric solutions, while the values of the deformation energy curves along the triaxial fission path are given in red. One can see that by allowing for triaxial deformation the fission barrier heights are reduced by $1-4 \mathrm{MeV}$ as compared with axially symmetric solutions. This lowering depends on the proton and neutron numbers. It also brings in average the results of the calculations in closer agreement with experimental data shown by green solid circles in Fig. 2. These circles display the height of the experimental fission barrier 12 at the calculated $\beta$-deformation of the saddle point. A typical uncertainty in the experimental values, as suggested by the differences 


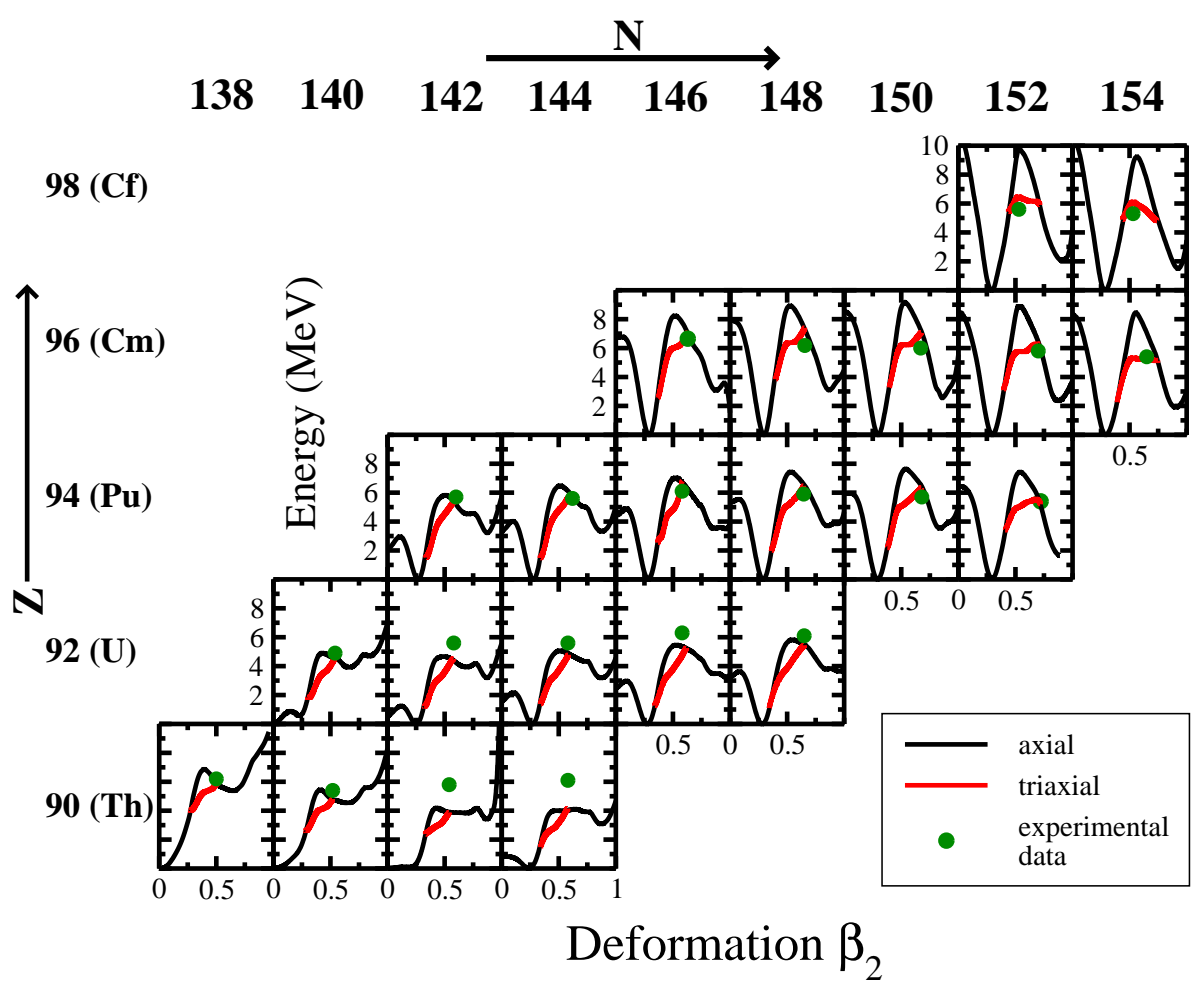

Fig. 2. (Color online) Deformation energy curves of even-even actinide nuclei obtained in the $\mathrm{RMF}+\mathrm{BCS}$ calculations with the NL3* parametrization. Black solid lines display the deformation energy curves for the axially symmetric solution, while red solid lines show the deformation energy curves along the triaxial part of the fission path. Solid green circles show the experimental values for the height of inner fission barrier.

among various compilations, is of the order of $\pm 0.5 \mathrm{MeV}$.

The microscopic origin of the lowering of the barrier due to triaxiality can be traced back to the changes of the level density in the vicinity of the Fermi level induced by triaxiality. The single-particle level density at the Fermi level is lower for triaxial solutions than for axially symmetric solutions. This is especially important at the deformation corresponding to the saddle point of the axially symmetric solution. Fig. 3] shows the differences between calculated and experimental heights of inner fission barriers. The average deviation between theory and experiment is 0.76 $\mathrm{MeV}$. This is comparable with the results obtained in the macroscopic + microscopic method.

\section{Microscopic theory of quantum phase transitions.}

Applications of CDFT discussed so far are based on the mean field concept. However, this framework has its limitations. It is characterized by symmetry breaking. 

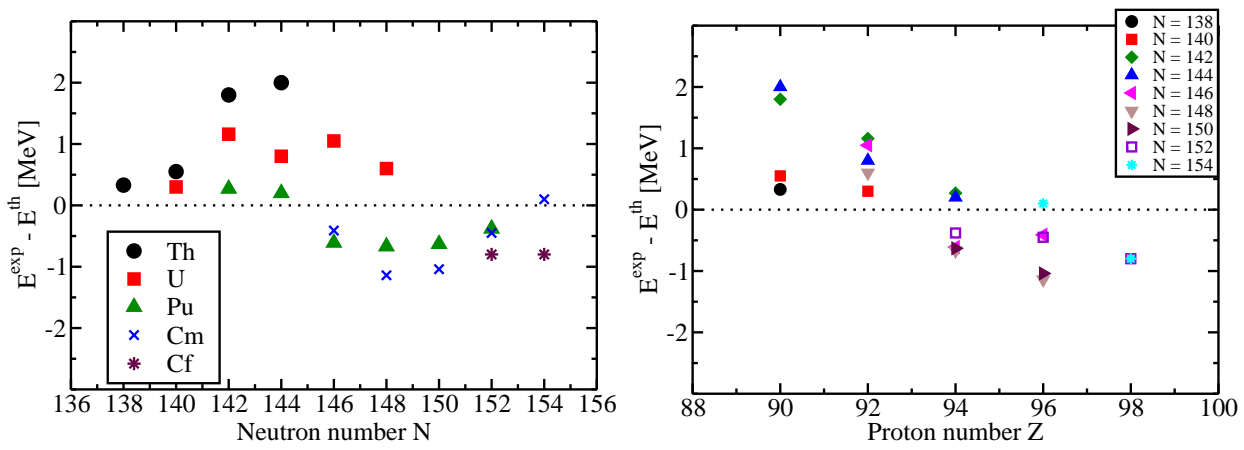

Fig. 3. (Color online) The difference between experimental and calculated heights of inner fission barriers as a function of neutron number $N$ and proton number $Z$

and can only provide an approximate description of specific nuclear excitations, such as collective rotations in the framework of the Cranking model or vibrations in the framework of time-dependent mean field theory, which corresponds in the small amplitude limit to RPA or QRPA. To calculate excitation spectra with specific quantum numbers and electromagnetic transition rates in individual nuclei, it is necessary to extend this framework to include collective correlations related to the restoration of broken symmetries and to fluctuations of collective coordinates. Collective correlations are sensitive to shell effects, display pronounced variations with particle number, and cannot be incorporated in a single Slater determinant.

On this level an extension of density functional theory beyond mean field takes into account collective correlations through the restoration of broken symmetries and configuration mixing of symmetry-breaking product states. In recent years several accurate and efficient models and algorithms have been developed that perform the restoration of symmetries broken by the static nuclear mean field, and take into account fluctuations around the mean-field minimum. The most effective approach to configuration mixing calculations is the generator coordinate method $(\mathrm{GCM})^{13}$ based on the variational ansatz for the many-body wave function:

$$
\left|\Psi_{\alpha}\right\rangle=\int d q f_{\alpha}(q) P^{I} P^{N} P^{Z}|\Phi(q)\rangle .
$$

The set of intrinsic wave functions $|\Phi(q)\rangle$ is generated by self-consistent constraint RMF+BCS calculations. The weight functions $f_{\alpha}(q)$ in the collective wave function of Eq. (11) are determined from the variation of the energy by the Hill-Wheeler equation:

$$
\int d q^{\prime}\left[\mathcal{H}\left(q, q^{\prime}\right)-E_{\alpha} \mathcal{N}\left(q, q^{\prime}\right)\right] f_{\alpha}\left(q^{\prime}\right)=0,
$$

where $\mathcal{H}$ and $\mathcal{N}$ are the angular-momentum projected GCM kernel matrices of the Hamiltonian and the norm, respectively.With the simplifying assumption of axial 
symmetry, GCM configuration mixing of angular-momentum, and even particlenumber projected quadrupole-deformed mean-field states, has become a standard tool in nuclear structure studies with non-relativistic $\frac{14 \mid 15}{}$, and relativistic density functionals 1617 .

Much more involved and technically difficult is the description of intrinsic quadrupole modes including triaxial deformations. Only very recently fully microscopic three-dimensional calculations have been presented for Skyrme ${ }^{18}$ and Gogny 19 mean field models generated by triaxial quadrupole constraints that are projected and mixed by the generator coordinate method. This method is actually equivalent to a seven-dimensional GCM calculation, mixing all five degrees of freedom of the quadrupole operator and the gauge angles for protons and neutrons. Only very recently $3 \mathrm{D}$ angular momentum projection 20 and configuration mixing of angular-momentum projected triaxial relativistic mean-field wave functions $20 \mid 21$ has been possible. However, triaxial calculations of this type are connected with an extreme numerical effort and are, at present, not possible for heavy nuclei.

Here we report on an axially symmetric application, where the set $|\Phi(q)\rangle$ is generated by imposing constraints on the axial $q_{20}$ mass quadrupole moments in self-consistent RMF+BCS calculations. The following calculations are based on the relativistic point coupling model $\mathrm{PC}-\mathrm{F} 122$. Pairing correlations are taken into account in the simple BCS approximation, using a $\delta$-pairing force.

Quantum phase transitions from spherical to deformed shapes have recently gained considerable interest. However, most of these investigations have been carried out within simple boson models 23 . In order to understand the microscopic background of such investigations in finite nuclei the relativistic Generator Coordinate Method has recently been applied 24 . Starting from constrained self-consistent mean-field calculations of potential energy curves, the one-dimensional generator coordinate method (GCM) has been used to perform configuration mixing of angularmomentum and particle-number projected relativistic wave functions 24 . It has been shown that the microscopic framework based on universal density functionals, adjusted only to nuclear ground-state properties and extended to take into account correlations related to symmetry restoration and fluctuations of collective variables, is able to describe not only general features of such shape transitions but also the unique behavior of excitation spectra and transition rates at the critical points. In particular the shape phase transition in the chain of $\mathrm{Nd}$ isotopes occurs precisely at the isotope ${ }^{150} \mathrm{Nd}$, in agreement with empirical evidence for the realization of the $\mathrm{X}(5)$ symmetry in the $\mathrm{N}=90$ rare-earth isotones. As shown in Fig. 4 the calculated spectrum and the intra-band and inter-band B(E2) transitions for ${ }^{150} \mathrm{Nd}$ are in excellent agreement with data 25 and close to the $\mathrm{X}(5)$-model predictions for the nucleus ${ }^{150} \mathrm{Nd} 23$.

As discussed before, because of the considerable numerical effort 3D angular momentum projected GCM calculations are at present not yet possible for such heavy nuclei as the Nd-chain. Therefore this phase transition has been investigated recently 26 on the basis of a 5 -dimensional Bohr Hamiltonian, whose parameters 


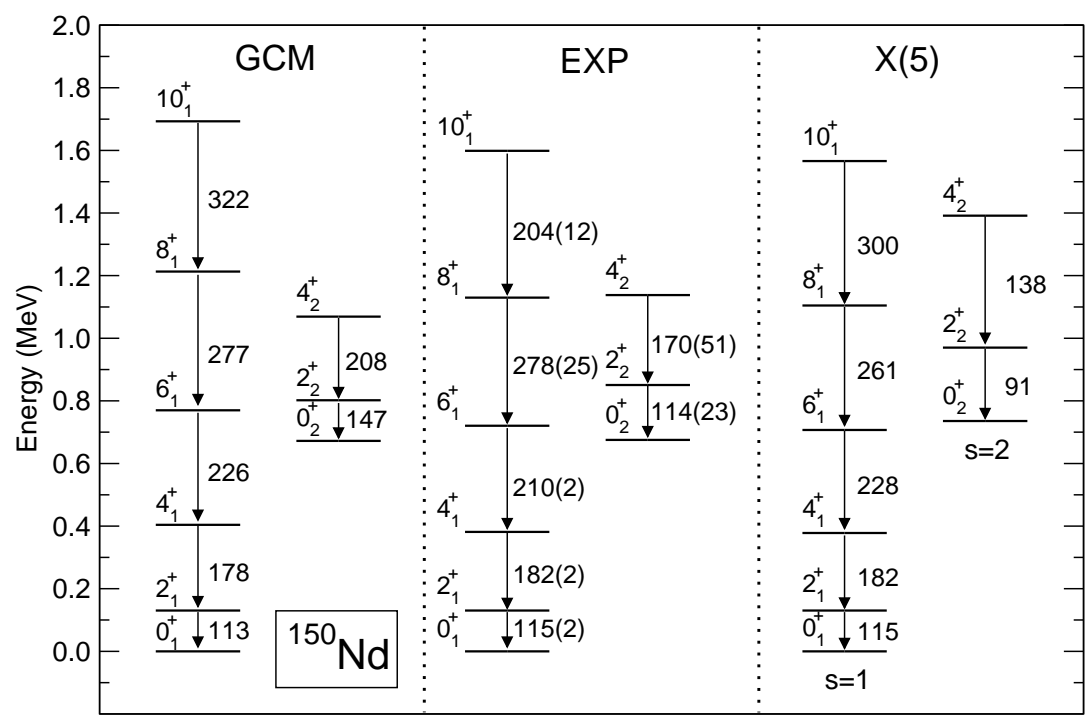

Fig. 4. The particle-number projected GCM spectrum of ${ }^{150} \mathrm{Nd}$ (left), compared with the and the $\mathrm{X}(5)$-symmetry predictions (right) for the excitation energies, and $\mathrm{B}(\mathrm{E} 2)$ values (in Weisskopf units) of the ground-state and the $\beta$-band. The theoretical spectra are normalized to the experimental energy of the state $2_{1}^{+}$, and the $\mathrm{X}(5)$ transition strengths are normalized to the experimental $\mathrm{B}\left(\mathrm{E} 2 ; 2_{1}^{+} \rightarrow 0_{1}^{+}\right)$.

were derived microscopically form the covariant density functional PC-F1. The resulting spectrum and the electromagnetic transition probabilities are more or less the same as in the 1D-AMP GCM calculation shown in Fig. 4.

\section{Summary and outlook.}

Among the microscopic approaches to the nuclear many-body problem, the framework of nuclear density functional theory provides the most complete description of ground-state properties and collective excitations over the whole nuclide chart. Here we have seen that the self-consistent relativistic mean-field model based on a universal density functional provides an excellent description of the inner fission barriers in actinide nuclei with an average deviation between theory and experiment of $0.76 \mathrm{MeV}$. For transitional nuclei relativistic models not only describe general features of shape transitions but also particular properties of spectra and transition rates at the critical point of the QPT. However, to calculate excitation spectra and transition probabilities, the self-consistent mean-field approach has to be extended to include correlations related to restoration of broken symmetries and fluctuations of collective variables. This can be done either by performing GCM 
configuration mixing calculations of projected wave functions or by constructing collective Bohr-type Hamiltonians with deformation-dependent parameters determined from self-consistent mean-field calculations. The possibility to perform selfconsistent microscopic studies of shape transitions opens a new perspective on the origin of nuclear quantum phase transitions in various mass regions. It is therefore important to systematically analyze these methods, also employing different energy-density functionals, various types of shape phase transitions that have been predicted in several regions of medium-heavy and heavy nuclei.

\section{Acknowledgments}

This work was partly supported by the DFG cluster of excellence "Origin and Structure of the Universe" (www.universe-cluster.de).

\section{References}

1. M. Bender, P.-H. Heenen, and P.-G. Reinhard, Rev. Mod. Phys. 75, 121 (2003).

2. P. Ring, Prog. Part. Nucl. Phys. 37, 193 (1996).

3. D. Vretenar, A. V. Afanasjev, G. A. Lalazissis, and P. Ring, Phys. Rep. 409, 101 (2005).

4. A. Sobiczewski and K. Pomorski, Prog. Part. Nucl. Phys. 58, 292 (2007).

5. A. Staszczak et al., Phys. Rev. C80, 014309 (2009).

6. M. Warda et al., Phys. Rev. C66, 014310 (2002).

7. S. Karatzikos et al., Phys. Lett. B689, 72 (2010).

8. M. Bender, K. Rutz, P.-G. Reinhard, J. A. Maruhn, and W. Greiner, Phys. Rev. C58, 2126 (1998).

9. T. Bürvenich et al., Phys. Rev. C69, 014307 (2004).

10. H. Abusara, A. V. Afanasjev, and P. Ring, Phys. Rev. C82, 044303 (2010).

11. G. A. Lalazissis et al., Phys. Lett. B671, 36 (2009).

12. M. Samyn, S. Goriely, and J. M. Pearson, Phys. Rev. C72, 044316 (2005).

13. P. Ring and P. Schuck, The Nuclear Many-Body Problem (Springer-Verlag, Berlin, 1980).

14. A. Valor, P.-H. Heenen, and P. Bonche, Nucl. Phys. A671, 145 (2000).

15. R. Rodríguez-Guzmán, J. L. Egido, and L. M. Robledo, Nucl. Phys. A709, 201 (2002).

16. T. Nikšić, D. Vretenar, and P. Ring, Phys. Rev. C73, 034308 (2006).

17. T. Nikšić, D. Vretenar, and P. Ring, Phys. Rev. C74, 064309 (2006).

18. M. Bender and P.-H. Heenen, Phys. Rev. C78, 024309 (2008).

19. T. R. Rodriguez and J. L. Egido, Phys. Rev. C81, 064323 (2010).

20. J. M. Yao, J. Meng, P. Ring, and D. Vretenar, Phys. Rev. C81, 044311 (2010).

21. J. M. Yao, H. Mei, H. Chen, J. Meng, P. Ring, and D. Vretenar, arXiv:1006.1400v1 [nucl-th] (2010).

22. T. Bürvenich et al., Phys. Rev. C65, 044308 (2002).

23. F. Iachello, Phys. Rev. Lett. 87, 052502 (2001).

24. T. Nikšić, D. Vretenar, G. A. Lalazissis, and P. Ring, Phys. Rev. Lett. 99, 092502 (2007).

25. R. Kruecken et al, Phys. Rev. Lett. 88, 232501 (2002).

26. Z. P. Li, T. Nikšić, D. Vretenar, J. Meng, G. A. Lalazissis, and P. Ring, Phys. Rev. C79, 054301 (2009). 